\title{
Socio-cultural practices, eating patterns and presence of double burden of malnutrition in mothers and their children in Grand-popo, Benin
}

\section{Abstract}

The world faces today a double burden of malnutrition which includes both undernutrition and overweight, especially in developing countries. Managing these two aspects of malnutrition in developing countries is a heavy burden for their failing health systems. Data from FAO show that 161 million children under five years are stunted and at the same time 3.4 million people die each year due to overweight and obesity (FAO, 2013). The aim of this study is to investigate the sociocultural influence, feeding practices and the presence of double burden of malnutrition in children and their mothers in Grand-popo's community in Benin. A cross-sectional, descriptive and analytical approach was used. A sample of 408 children between 0- 59 months and their mothers were selected. A questionnaire was developed to get information on demographic and socio-economic status of the household, breastfeeding and infant feeding practices. Food frequency questionnaire was administrated to assess dietary patterns and four focus groups with mothers were held to determine care practices and food taboos. Weight and height measurements for children and weight, height and waist circumference for their mothers were taken. Height for Age and Weight for Height z scores were determined for children and body mass index (BMI) measurements were calculated for women. For statistics analysis, IBM SPSS 25 was used. The mean age of children was $24.45 \pm 14.9$ months with $53.2 \%$ of girls in the sample. The mean age of mothers was $27.99 \pm 6.99$ years. Majority of mothers $(93.1 \%)$ are married or living with the spouse. About $91.7 \%$ of household had a monthly income of $\$ 60$ and spent on average $\$ 24 \pm \$ 11.2$ on food. Prevalence of wasting was $9.8 \%$ and stunting $29.7 \%$. Waist circumference mean of mothers was $79.95 \pm$ $10.35 \mathrm{~cm}$ and $19.3 \%$ were classified to be at high metabolic risk. BMI assessments revealed $16.9 \%$ of mothers were overweight and $7.4 \%$ obese. All children were breastfed. Children were introduced to family foods at 5 months on average with very low consumption of animal protein and fruits, and a high consumption of vegetables and maize based meals. Food taboos particularly during pregnancy were revealed and those cultural beliefs were still followed by some mothers. The findings showed the need for more effort on cultural sensitive interventions to reduce double burden of malnutrition.

\section{Conflict of Interest}

There is no conflict of interest. 